\title{
Endoscopic Ultrasound-Guided Hepaticogastrostomy: Technical Review and Tips to Prevent Adverse Events
}

\author{
Takeshi Ogura and Kazuhide Higuchi \\ 2nd Department of Internal Medicine, Osaka Medical College, Osaka, Japan
}

Article Info
Received March 11, 2020
Revised April 14, 2020
Accepted May 8, 2020
Published online July 23, 2020
Corresponding Author
Takeshi Ogura
ORCID https://orcid.org/0000-0003-2916-6568
E-mail oguratakeshi0411@yahoo.co.jp

E-mail oguratakeshi0411@yahoo.co.jp

\begin{abstract}
Most patients who require biliary drainage can be treated by endoscopic retrograde cholangiopancreatography (ERCP)-guided procedures. However, ERCP can be challenging in patients with complications, such as malignant duodenal obstruction, or a surgically-altered anatomy, such as a Roux-en-Y anastomosis, which prevent advancement of the duodenoscope into the ampulla of Vater. Recently, endoscopic ultrasound (EUS)-guided biliary drainage via transhepatic or transduodenal approaches has emerged as an alternative means of biliary drainage. Typically, EUS-guided gallbladder drainage or choledochoduodenostomy can be performed via both approaches, as can EUS-guided hepaticogastrostomy (HGS). EUS-HGS, because of its transgastric approach, can be performed in patients with malignant duodenal obstruction. Technical tips for EUS-HGS have reached maturity due to device and technical developments. Although the technical success rates of EUS-HGS are high, the rate of adverse events is not low, with stent migration still being reported despite many preventive efforts. In this review, we described technical tips for EUS-HGS related to bile duct puncture, guidewire insertion, fistula dilation, and stent deployment, along with a literature review. Additionally, we provided technical tips to improve the technical success of EUS-HGS. (Gut Liver 2021;15:196-205)
\end{abstract}

Key Words: Endoscopic retrograde cholangiopancreatography; Endoscopic ultrasound-guided biliary drainage; Drainage; Endoscopic ultrasound

\section{INTRODUCTION}

Malignant biliary obstruction is usually treated under endoscopic retrograde cholangiopancreatography (ERCP) guidance. ${ }^{1,2}$ Most patients who need biliary drainage can be treated using ERCP guidance. ${ }^{3}$ However, ERCP can be challenging in patients with complications, such as malignant duodenal obstruction, or a surgically-altered anatomy, such as a Roux-en-Y anastomosis, which prevent advancement of the duodenoscope into the ampulla of Vater. Percutaneous transhepatic biliary drainage (PTBD) has been conventionally attempted as an alternative to bile duct drainage. Biliary drainage can also be achieved under single-balloon enteroscopy (SBE) or double-balloon enteroscopy $(\mathrm{DBE})^{4-6}$ in patients with surgically-altered anatomy. However, although these procedures have clinical benefits, their disadvantages include self-tube removal or cosmetic issues after PTBD, and prolonged procedures and risk of perforation in SBE or DBE.

Endoscopic ultrasound-guided biliary drainage (EUSBD) via transhepatic or transduodenal approaches has recently emerged as an alternative means of biliary drainage. Typically, EUS-guided gallbladder drainage (GBD) or choledochoduodenostomy (CDS) can be performed via both approaches. Additionally, EUS-guided hepaticogastrostomy (HGS) can also be performed. Among these approaches, EUS-HGS, because of its transgastric approach, can be performed in patients with malignant duodenal obstruction. ${ }^{7}$ Initial EUS-HGS procedures were technically challenging and associated with critical adverse events such as stent migration. ${ }^{8}$ However, along with device and technical developments, the technical tips for EUS-HGS have also reached maturity. Despite this, however, while the technical success rates of EUS-HGS are currently high, the rate of adverse events is not low, with stent migration still being reported ${ }^{9-13}$ despite many preventive efforts. 
This review describes technical tips to improve the technical success of EUS-HGS, and provides a literature review of adverse event prevention.

\section{SUMMARY OF REPORTS RELATED} TO EUS-HGS

Various studies and meta-analyses on EUS-HGS have been published. Table 1 shows reports of EUS-HGS for malignant biliary obstruction that included over 10 cases. $^{14-39}$ The reported technical and clinical success rates of EUSHGS ranged from $65 \%$ to $100 \%$, and from $76 \%$ to $100 \%$, respectively. The most frequent adverse events were bleeding $(n=12)$, followed by bile peritonitis $(n=8)$, bile leak $(n=7)$, and pneumoperitoneum $(n=4)$. In addition, stent migration was observed in four patients. Patient death due to procedural related adverse events occurred in four patients. The standard algorithm for performing EUS-HGS in patients with advanced malignant biliary obstruction at our hospital is presented in Fig. 1. Since duodenal obstruction is one of the risk factors for early biliary stent obstruction, EUS-HGS is considered as the first option in patients with duodenal obstruction. Of course, if ERCP fails, an EUS-guided rendezvous technique is attempted. EUSHGS has several disadvantages. First, as shown in Table 1, adverse events are not infrequent. Second, stent migration is sometimes fatal, and although bile peritonitis is usually treated conservatively, this adverse event might be critical in patients with advanced malignant tumor. Therefore, endoscopists should carefully consider the indications for EUS-HGS. In patients with massive ascites, severe adverse events, such as stent migration, can occur. In addition, in patients with insufficient dilatation of the intrahepatic bile duct, bleeding can occur while puncturing the bile duct because blood vessels usually run near the intrahepatic bile duct.

\section{TECHNICAL TIPS FOR BILE DUCT PUNCTURING}

The first step in EUS-HGS is puncture of the intrahepatic bile duct using a 19-gauge fine needle. Two important points should be focused on here. One is the puncture site, and the other is avoidance of vessel puncture, which can be quite simply achieved using color Doppler ultrasound.

No evidence has been published about which of the bile duct sites B2, or B3, is more suitable for EUS-HGS. Generally, guidewire manipulation is easier if B2 is punctured rather than $\mathrm{B} 3$ because the bile duct runs relatively straight. However, if B2 is punctured, a puncture site at the intraluminal portion might be from the esophagus. Therefore, severe adverse events such as mediastinitis, can occur. ${ }^{7}$ If puncture site is not performed via the esophagus, the intraluminal puncture site is sometimes located in the stomach directly below the esophagus. In this situation, the proximal portion of the EUS-HGS stent might turn in the oral direction during deployment. Severe adverse events such as these have been associated with EUS-HGS stents. ${ }^{40}$ Therefore, we recommend puncture at the B3 site during EUS-HGS. With B3 puncture, the echoendoscope angle might be helpful in preventing puncture from the esophagus. Fig. 2 shows that the angle of the echoendoscope might be approximately $170^{\circ}$ in the esophagus due to the limited width of the esophagus, but around $90^{\circ}$ when it is in the stomach. Therefore, the shape of the scope should be checked by fluoroscopic imaging before intrahepatic bile duct puncture. The scope shape might also be important for guidewire manipulation. In addition, the bile duct, which runs from the upper left to the lower right corner on EUS images should be punctured to advance the guidewire towards the hepatic hilum.

\section{TECHNICAL TIPS FOR GUIDEWIRE} MANIPULATION

Guidewire manipulation is the most limiting step during EUS-HGS, particularly when attempted by nonexperts. Guidewire shearing can occur under awkward guidewire manipulation. Vila et al. ${ }^{16}$ have described their initial experience with interventional EUS in a multicenter, retrospective, national survey of 125 patients in Spain who underwent interventional EUS. Among them, EUSguided puncture was achieved in 120 patients, and contrast medium was injected into the ducts of 113 patients. However, unsuccessful manipulation of the guidewire was responsible for $68.2 \%$ of the technical failures. Therefore, they stated that intraductal manipulation of the guidewire seems to be the most challenging stage of the procedure. Guidewire manipulation can fail during insertion into the intrahepatic bile duct through the fine-needle aspiration (FNA) needle and while advancing the guidewire into the hepatic hilum after inserting it into the intrahepatic bile duct.

When the guidewire is inserted into the intrahepatic bile duct, it can sometimes penetrate the hepatic parenchyma (Fig. 3A). The guidewire should be gently manipulated to prevent this. Appropriate guidewire selection is also important. A flexible tip on the guidewire might be important, and the knuckle guidewire technique might be 


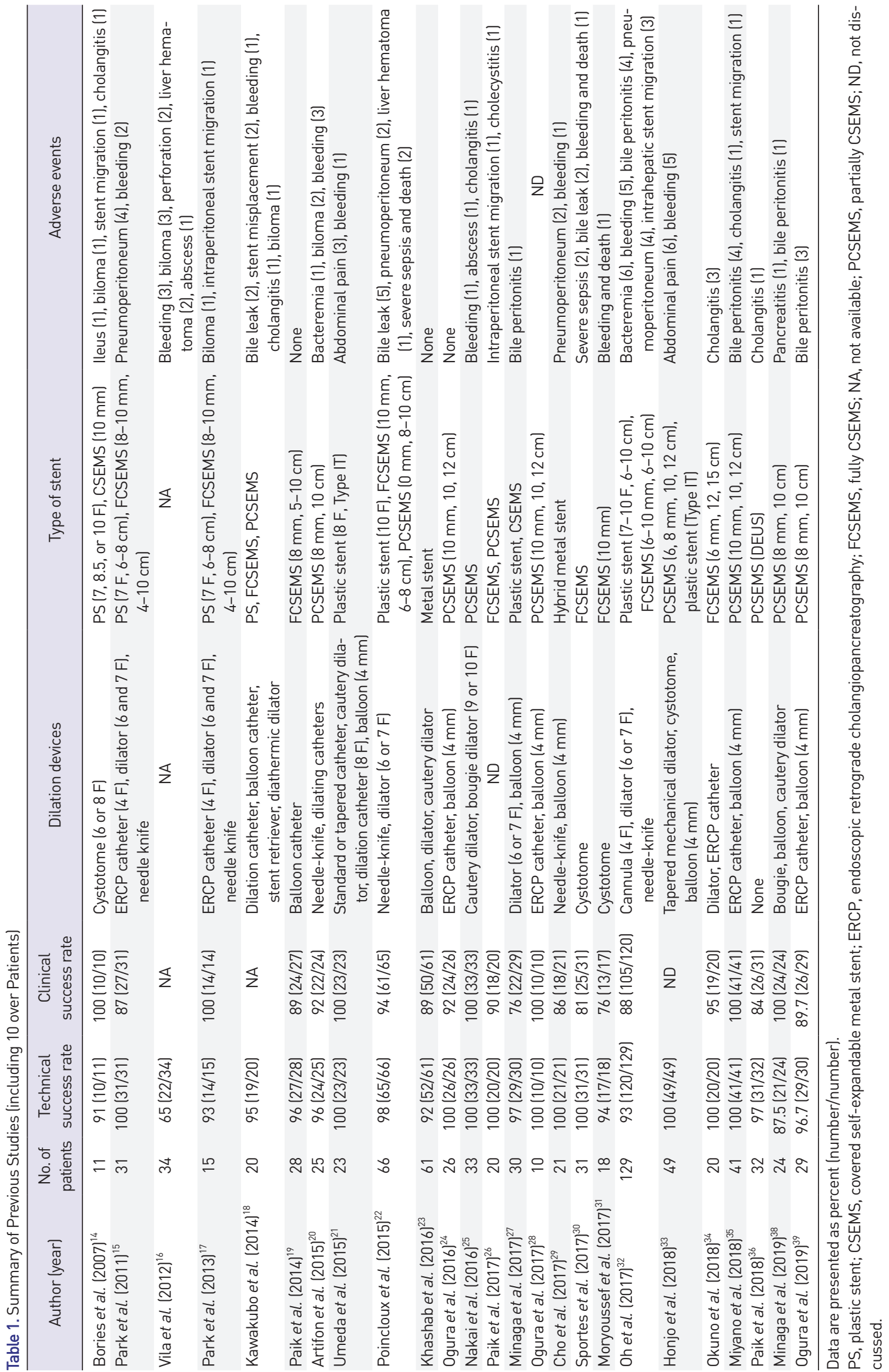


helpful. ${ }^{41}$ Therefore, guidewire selection should be decided based on flexibility of the tip of the guidewire. However, endoscopists should also consider stiffness, because several devices, such as dilation devices and the stent delivery system need to be inserted after guidewire deployment.

If the guidewire is advanced into the hepatic parenchyma (Fig. 3B) or into the periphery of the bile duct (Fig. 3C), the guidewire should be manipulated towards the hepatic hilum again. Guidewire shearing can also occur during this procedure. Fig. 4 shows that while guidewire shearing can easily occur if the angle between the FNA needle and the guidewire is acute, it can even occur when it is obtuse. The echoendoscope angle might help to achieve successful guidewire insertion. If the scope angle is obtuse, the angle between the bile duct and FNA needle might be acute, and vice versa. Therefore, the intrahepatic bile duct should be punctured after checking the scope shape on fluoroscopic images. However, despite these techniques, the guidewire can still advance to the periphery of the bile duct. In this situation, the guidewire should be withdrawn a little and redirected towards the hepatic hilum. The liver impaction technique might be helpful during this step, as shown in Fig. $5 .{ }^{42}$ When the FNA needle is pulled into

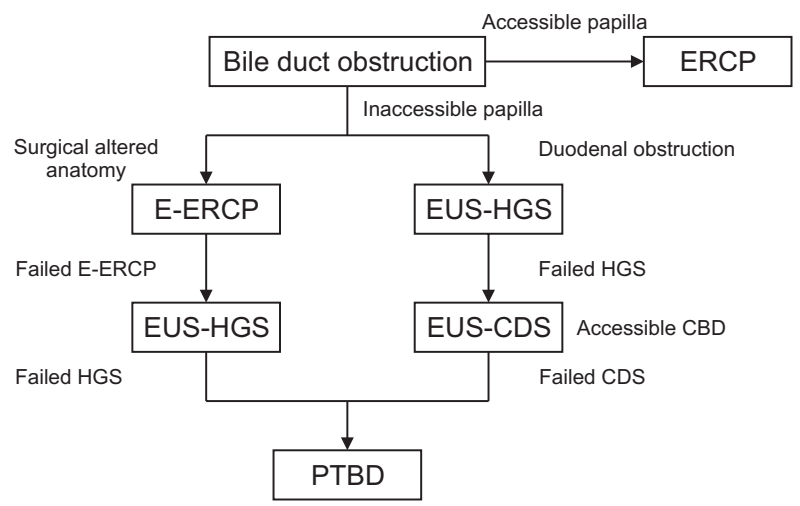

Fig. 1. Drainage algorithm used at our hospital.

CBD, common bile duct; CDS, choledochoduodenostomy; ERCP, endoscopic retrograde cholangiopancreatography; E-ERCP, enteroscopy-guided ERCP; EUS, endoscopic ultrasound; HGS, hepaticogastrostomy; PTBD, percutaneous transhepatic biliary drainage. the hepatic parenchyma, the guidewire can be easily manipulated due to the following reasons: a tamponade effect around the tip of the FNA needle, and fact that the angle between the FNA needle and the guidewire might prevent guidewire shearing. If the FNA needle is pulled, the angle between the guidewire will increase.

A novel steerable access device for EUS-BD has recently become available. ${ }^{43}$ Ryou et al. reported their initial clinical experience with this device for EUS-BD. The device has a sharp stylet to allow for puncture. After stylet removal, the blunt-tipped access catheter (18.5 gauge) assumes a predetermined curvature $\left(90^{\circ}\right.$ or $\left.135^{\circ}\right)$ and is fully rotatable. The curvature is predetermined by fenestrations at the catheter tip. Guidewire sharing is theoretically avoided because of the blunt tip and the coaxial orientation of the wire relative to the catheter tip. Although, so far, only one patient has undergone EUS-HGS using this device, it shows promise in preventing guidewire shearing.

\section{TECHNICAL TIPS FOR FISTULA DILATION}

To fit the axis of the puncture route to insert another device might be technically important. Fig. 6 shows that accurate fitting of the axis allows clear visualization of all devices, and that the echoendoscope angle should be essentially the same as that before puncture and insertion of
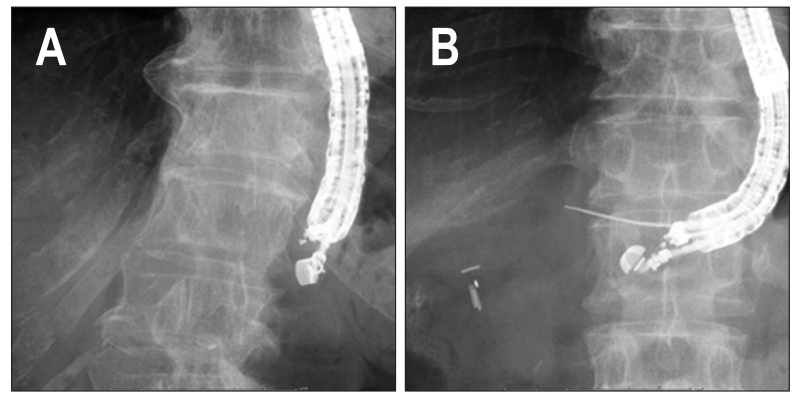

Fig. 2. (A) The angle of the echoendoscope in the esophagus is approximately $170^{\circ}$ due to the limited width of the esophagus. (B) The angle of the echoendoscope is approximately $90^{\circ}$ if it is in the stomach.
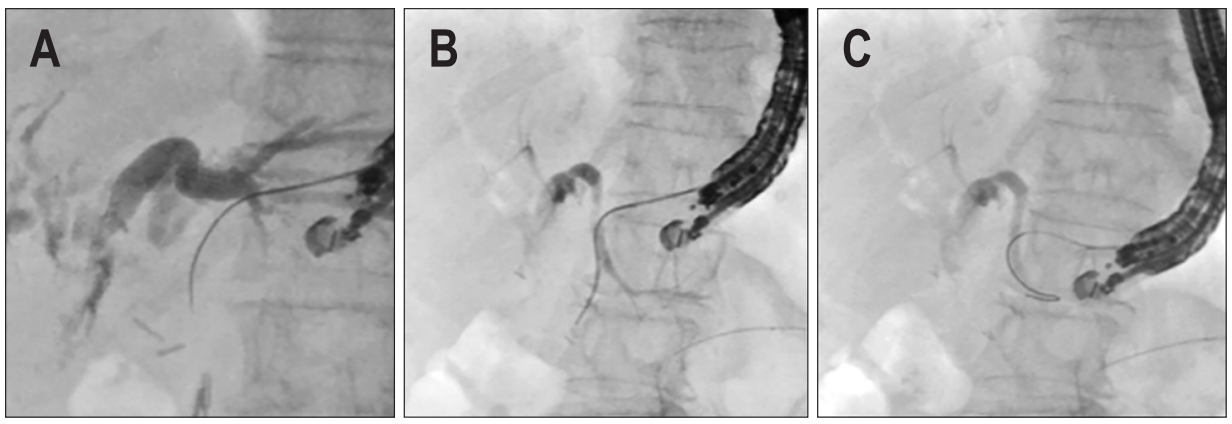

Fig. 3. (A) The guidewire penetrates the hepatic parenchyma. (B) The guidewire is inserted into the branch bile duct. (C) The guidewire is advanced into the periphery of the bile duct. 
the stent delivery system. If the device fits the axis, it would facilitate devices with a relatively large diameter to be easily inserted into the bile duct.

Various dilation techniques have been described to
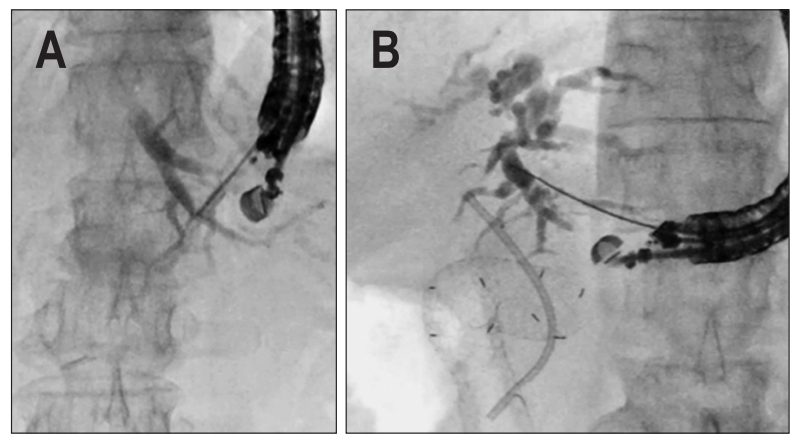

Fig. 4. (A) If the scope angle is obtuse, the angle between the bile duct and fine-needle aspiration (FNA) needle might be acute. (B) If the scope angle is acute, the angle between the bile duct and FNA needle might be obtuse. achieve this. Honjo et al. ${ }^{33}$ compared dilation with an ultra-tapered mechanical $(n=26)$ or electrocautery $(n=23)$ dilator (control) during EUS-HGS. The technical success rate of tract dilation did not differ significantly between the groups (electrocautery vs mechanical dilation: $23 / 23$ [100\%] vs $24 / 26$ [92.3\%], $\mathrm{p}=0.52$ ), although the procedure duration was shorter in the electrocautery group $(17.5 \pm 5$ minutes vs $21.5 \pm 6.5$ minutes, $\mathrm{p}=0.09$ ). However, the adverse event of bleeding was significantly more frequent in the electrocautery group ( 5 vs $0, p=0.04$ ). Therefore, they concluded that the ultra-tapered mechanical dilator designed for interventional EUS was safe and useful as it reduced post-procedural bleeding, together with having a high technical success rate compared with conventional electrocautery. We previously described the clinical benefits of dilation using a novel fine gauge balloon catheter for EUS-BD ${ }^{44}$ in a prospective, single-center, single-arm study of 20 patients, among whom nine underwent EUSHGS. Technical success was defined as the insertion of a stent delivery system into the intrahepatic bile duct using
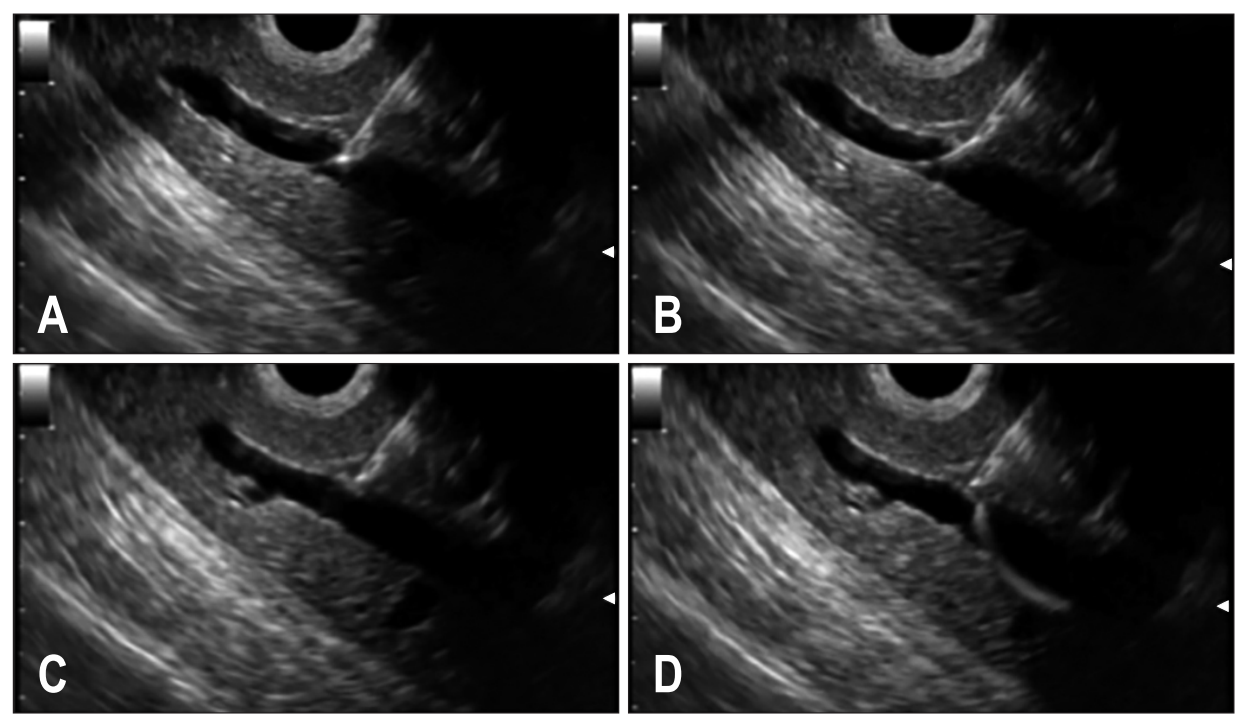

Fig. 5. Liver impaction technique. (A) The guidewire is inserted into the periphery of the bile duct. (B) The fine-needle aspiration (FNA) needle alone is pulled back into the hepatic parenchyma. (C) The guidewire is easily pulled into the FNA needle. (D) Guidewire manipulation is successfully performed without wire shearing.
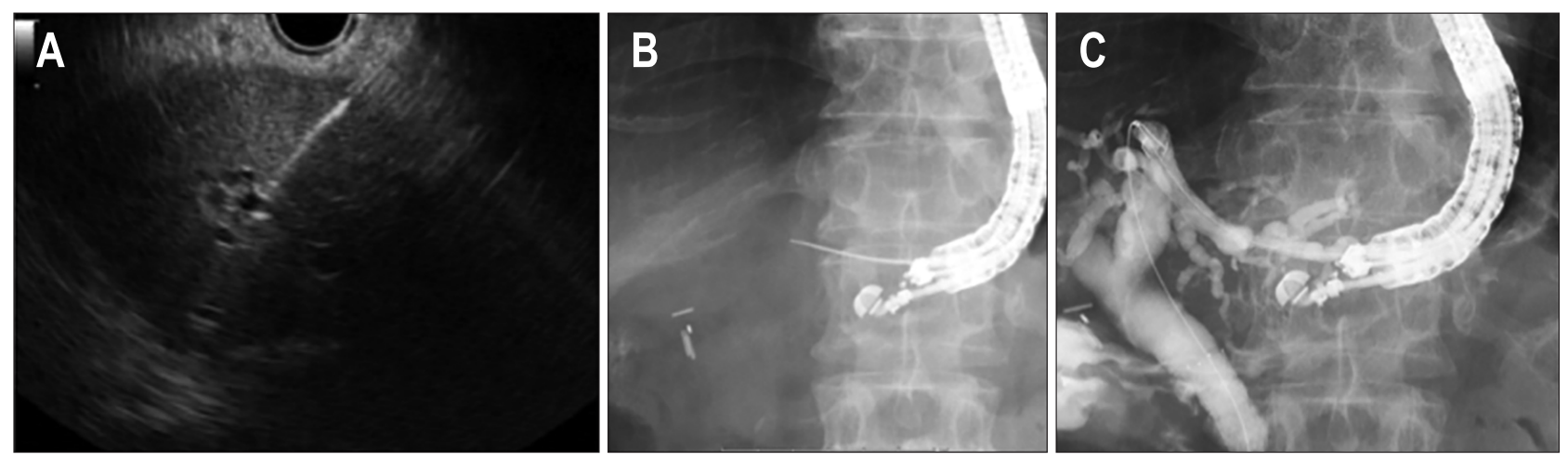

Fig. 6. Proper fitting of the axis allows clear visualization of all devices under endoscopic ultrasound (A); the echoendoscope angle after puncture should be essentially the same as that before puncture $(B)$; insertion of the stent delivery system $(C)$. 
a novel fine gauge balloon catheter without additional dilation. Consequently, all nine patients underwent EUSHGS within a median duration of 14 minutes. Therefore, we concluded that our technique might facilitate EUS-BD. However, data from more patients and a randomized controlled comparison with other dilation techniques, such as graded or cystotome dilation, are needed.

On the other hand, electrocautery dilation might be perceived as risky, according to previous reports. Park et al. ${ }^{15}$ evaluated risk factors for adverse events associated with EUS-BD in 57 consecutive patients with malignant or benign biliary obstruction treated by EUS-BD after failed ERCP. That study significantly associated post-procedure adverse events with fistula dilation using a needle-knife compared with graded dilation $(9 / 27$ [33\%] vs $2 / 28$ [7\%], $\mathrm{p}=0.02$ ). Therefore, they recommended avoiding this practice in EUS-BD. The adverse event of bleeding might occur due to the "burn effect." Indeed, a comparison of electrocautery and mechanical dilation ${ }^{33}$ found no significant difference in terms of technical success rates and the number of additional dilations of the needle tract required to accomplish stent placement. In addition, procedurerelated bleeding developed in six (18\%) patients with electrocautery dilation, and in none during mechanical dilation $(\mathrm{p}=0.04)$. These results showed that the mechanical dilator allows safe tract dilation without the "burn effect" regardless of procedure. However, intrahepatic bile duct or stomach wall dilation can be difficult with non-electrocautery dilation devices in clinical practice, especially in patients with frequent cholangitis. Electrocautery dilation might be necessary in such patients. A novel fine gauge electrocautery dilator has recently become available in Japan. ${ }^{45}$ The distal end of the outer dilator has a metal tip that is only $3 \mathrm{~F}$ at the top, and the dilator is guided with a coaxial 0.025 -inch guidewire. Therefore, the prevalence of the "burn effect" might be lower with this device than with conventional electrocautery dilation. In that study, EUS$\mathrm{BD}$, including EUS-GBD, CDS and HGS without dilation devices, were successful in all patients. In addition, severe adverse events such as bleeding did not occur. Although more patients and a prospective evaluation are essential to confirm these results, the fine gauge electrocautery dilator has the potential to improve the outcomes of EUS-BD.

Bile can leak from the intrahepatic bile duct after fistula dilation. Therefore, this step should be straightforward, and the procedural duration should be shortened or even omitted to decrease the likelihood of adverse events. According to this viewpoint, one-step stent deployment might be ideal. Park et al. ${ }^{46}$ evaluated the feasibility and safety of a novel dedicated device for one-step EUS-BD in a randomized trial of 32 patients with malignant biliary obstruction and failed ERCP. The patients were assigned to undergo the procedure in which a dedicated stent introducer was used with a modified hybrid metal stent (DH group, $\mathrm{n}=16$ ) or a conventional 8.5-F biliary metal stent introducer was used with a fully covered metal stent (FC group, $n=16$ ). Technical success was defined as stent deployment without a dilation device in the DH group. One-step technical success was achieved in 14 (88\%) of 16 patients, and the procedure was significantly shorter in the $\mathrm{DH}$ group (10 minutes vs 15 minutes, $\mathrm{p}=0.007)$. The rate of early adverse events was lower in the $\mathrm{DH}$ group (6.3\% vs $31.3 \%, \mathrm{p}=0.172$ ), although the difference did not reach statistical significance. Hence, although this stent might confer benefits, since the onestep technical success rate was not $100 \%$, further improvement of the one-step stent delivery system is needed.

\section{STENT SELECTION AND TECHNICAL TIPS FOR STENT DEPLOYMENT}

Critical adverse events, such as stent migration, can occur during this step. ${ }^{8-13}$ Previous reports indicate that almost all endoscopists use fully (FCSEMS) or partially (PCSEMS) covered self-expandable metal stents. The latter might have several advantages over the former. Since the site at the distal side is uncovered, branch bile duct obstruction might be prevented. If branch bile duct obstruction does occur as a complication, focal cholangitis can occur at the obstruction site. Uncovered sites of the PCSEMS might also play a role in anchoring the stent, which might prevent stent dislocation into the stomach. However, this needs to be confirmed in a prospective randomized controlled study. New metal stents dedicated to EUS-HGS have recently been developed. Park et al. ${ }^{46}$ investigated the feasibility of a new device for one-step EUS-BD, in which the stent has a dedicated introducer with a 3-F catheter and 4-F tapered metal tip. Therefore, simple puncture of the intestinal wall and hepatic parenchyma can be achieved without the need for additional dilation. This stent has an uncovered, funnel-shaped proximal end, a covered body, and a distal portion covered with a silicone membrane. The distal end is equipped with four flaps to prevent inward stent migration. Indeed, none of the stents migrated in that study. Cho et al. ${ }^{29}$ described the long-term outcomes of a new hybrid metal stent specifically for EUS-BD which has a $3.5-\mathrm{cm}$ distal portion comprising a silicone-covered nitinol wire to prevent bile leakage. In addition, the covered portion has proximal and distal anchoring flaps to immobilize the stent and prevent proximal and distal migration after placement. The proximal portion, which ranges from 1.5 to $6.5 \mathrm{~cm}$ in length, is uncovered to prevent intrahe- 
patic bile duct obstruction. The term "hybrid" refers to the two main features of the stent: anchoring flaps that reduce stent migration, and an uncovered portion that decreases the likelihood of obstructive cholangitis. The diameter and length of the hybrid stent are $8-10 \mathrm{~mm}$ and $5-10 \mathrm{~cm}$, respectively and the delivery system has a diameter of $8 \mathrm{~F}$. The technical success rate of EUS-HGS. using this stent in 21 patients was $100 \%$, and none of the stents migrated during the procedure or over a median follow-up period of 148.5 days. While these novel stents might positively impact clinical practice, stent migration has recently been reported. ${ }^{13}$ Therefore, the length of stents might also impact their migration. We recently evaluated factors associated with stent patency in EUS-HGS ${ }^{47}$ in 51 patients. Median stent patency was significantly shorter when the length of the luminal portion was $<3$ compared with $\geq 3 \mathrm{~cm}$ (52 days vs 195 days, $\mathrm{p}<0.01$ ). In contrast, median stent patency did not differ significantly between luminal portions $\geq 4$ and $<4 \mathrm{~cm}$ (194 days vs 127 days, $\mathrm{p}=0.1726$ ). A luminal portion $\geq 3 \mathrm{~cm}$ in length (hazard ratio [HR], 9.242; 95\% confidence interval [CI], 3.255 to $26.244 ; \mathrm{p}<0.05)$ and the performance of chemotherapy (HR, 3.022; 95\% CI, 1.448 to 6.304 , $\mathrm{p}<0.05$ ) were also associated with prolonged stent patency in Cox proportional hazards models. In addition, none of the stents migrated after EUS-HGS. Nakai et al. ${ }^{25}$ also described the safety and efficacy of EUS-HGS using a long PCSEMS (LP-CMS) for malignant biliary obstruction in 33 patients. Their study reported a $100 \%$ technical success rate with stent lengths of 10 and $12 \mathrm{~cm}$ in $76 \%$, and $24 \%$ of the patients, respectively. Although the adverse events of bleeding, abscess formation and cholangitis occurred in one patient each, none of the stents migrated either during the procedure or during follow-up.

While the type of metal stent selected is important to prevent migration, technical tips for stent deployment are also important. Technically inappropriate stent deployment can lead to stent migration during deployment. ${ }^{48}$ Intra-scope channel release is reportedly clinically useful. ${ }^{19,35}$ We previously used computed tomography to mea- sure distances between the hepatic parenchyma and the stomach wall in patient groups that underwent EUS-HGS with extra-scope $(n=20)$ and intra-scope $(n=21)$ channel release. The distance (mean \pm standard deviation) between the hepatic parenchyma and stomach wall was significantly shorter after intra-scope than extra-scope channel release $(0.66 \pm 1.25 \mathrm{~cm}$ vs $2.52 \pm 0.97 \mathrm{~cm})$. Therefore, although EUSHGS stents and stent deployment techniques require further improvement, intra-scope channel release and long PCSEMS might be important in preventing stent migration, especially when the procedure is being performed by non-expert endoscopists.

A new plastic stent has become available in Japan. ${ }^{21}$ It is a single-pigtail bile duct stent (total length, $20 \mathrm{~cm}$; effective length, $15 \mathrm{~cm}$; four flanges with apertures and side holes; total of 12 holes; distal straight part; four holes and a pigtail site; eight holes) with a tapered tip. Umeda et al. ${ }^{21}$ conducted a prospective preliminary feasibility study of EUS-HGS using this stent in 33 patients. The technical success rate was $100 \%$ without stent migration during a median followup period of 5.0 months, although several adverse events such as self-limiting abdominal pain, occurred. Therefore, they concluded that this new EUS-HGS-dedicated plastic stent was technically feasible and might be applicable in selected patients.

However, plastic stents have several disadvantages as compared to metal stents for EUS-HGS. The diameter of plastic stent is smaller, than that of metal stents, which might shorten the duration of stent patency. this would require discontinuation chemotherapy to treat recurrent biliary obstruction, which, in turn, would influence the survival or quality of life of patients. In addition, if a stent becomes occluded before fistula creation between the hepatic parenchyma and the stomach wall, reintervention might be challenging. Next, the tamponade effect is not obtained because plastic stents are not self-expandable. This function might play important roles in the prevention of bleeding or bile leakage from puncture sites or fistulae. Although these theories should be confirmed by
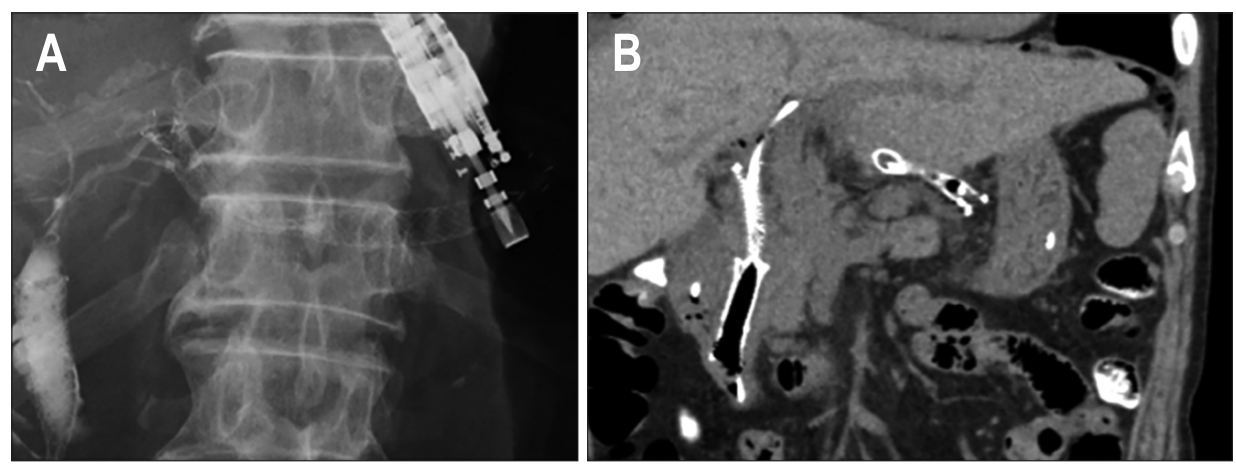

Fig. 7. (A) Endoscopic ultrasoundguided hepaticogastrostomy is performed. (B) Computed tomography image showing stent migration into the abdominal cavity. 
a randomized comparison of metal and plastic stents, we recommend EUS-HGS using metal stents. However, the risk of stent migration, which can lead to critical outcomes in patients, might be higher with these stents.

To overcome this adverse feature of metal stents, EUSHGS has been combined with antegrade stent (EUSHGAS) deployment, ${ }^{49}$ which has several advantages. Double stent deployment might prolong stent patency compared with EUS-HGS. This is because if one of the stents becomes occluded, recurrent biliary obstruction does not occur due to patency of the second stent. Next, because the antegrade stent is deployed before the EUSHGS, bile leakage through the fistula is unlikely. However, this feature is also seen if the antegrade stent is deployed without fistula dilation. Therefore, a metal stent with a fine gauge stent delivery system should be selected to realize this benefit. Additionally, if the stent migrates during EUS-HGS deployment, pressure on the biliary tract might be decreased due to the presence of the EUS-guided antegrade stenting (EUS-AS), and hence, conservative treatment might be enough. Indeed, we have experienced case of EUS-HGS stent migration in which conservative treatment alone was required because of the EUS-AS (Fig. 7).

\section{TECHNICAL TIPS FOR REINTERVENTION FOR OCCLUDED EUS-HGS STENTS}

Recently, overall survival in patients with hepatobiliary malignancy has been prolonged due to improvement of chemotherapy with drugs such as Folfirinox. Therefore, reintervention for EUS-HGS should be considered in cases with EUS-HGS stent occlusion. Reintervention for occluded EUS-HGS stents depends on the type of stents. If plastic stents are deployed, stent exchange might not be difficult. However, use of long SEMS for EUS-HGS might prevent stent migration. EUS-HGS stents might become occluded due to mucosal hyperplasia at the distal end of the EUSHGS stent or by the presence of sludge. However, owing to the long length of the stent in the gastric lumen, reintervention might be sometimes challenging. Several authors described their efforts with reintervention techniques. We previously described a simplified reintervention method for EUS-HGS stent obstruction. In our method, the covered site of the FCSEMS is first penetrated by use of a diathermic dilator. Next, an ERCP catheter is inserted and easily advanced into the intestine. Stent placement for the occluded HGS stent is also performed by use of an uncovered metal stent. Minaga et al. ${ }^{50}$ also described a similar technique using a precut needle knife. The most suitable technique requires evaluation by further clinical study.

\section{CONCLUSIONS}

The rate of adverse events during EUS-HGS is still high, and such events can sometimes be fatal, as with stent migration. Also, bile peritonitis might occur during fistula dilation. Therefore, one-step stent deployment without device exchanges is most ideal. Additionally, to prevent stent migration, improvements in stents, such as lumen apposing formation, is also required. Finally, endoscopists should pay attention not only to technical success, but also preventing adverse events, to improve the clinical benefits of EUS-HGS in the selected patients in whom it is performed.

\section{CONFLICTS OF INTEREST}

No potential conflict of interest relevant to this article was reported.

\section{ORCID}

Takeshi Ogura https://orcid.org/0000-0003-2916-6568

Kazuhide Higuchi https://orcid.org/0000-0002-4142-2272

\section{REFERENCES}

1. Fogel EL, Sherman S, Devereaux BM, Lehman GA. Therapeutic biliary endoscopy. Endoscopy 2001;33:31-38.

2. Williams EJ, Taylor S, Fairclough P, et al. Are we meeting the standards set for endoscopy? Results of a large-scale prospective survey of endoscopic retrograde cholangiopancreatograph practice. Gut 2007;56:821-829.

3. Tonozuka R, Itoi T, Tsuchiya T, Tanaka R, Mukai S. EUSguided biliary drainage is infrequently used even in highvolume centers of interventional EUS. Gastrointest Endosc 2016;84:206-207.

4. Shimatani M, Matsushita M, Takaoka M, et al. Effective "short" double-balloon enteroscope for diagnostic and therapeutic ERCP in patients with altered gastrointestinal anatomy: a large case series. Endoscopy 2009;41:849-854.

5. Yamada A, Kogure H, Nakai Y, et al. Performance of a new short-type double-balloon endoscope with advanced force transmission and adaptive bending for pancreaticobiliary intervention in patients with surgically altered anatomy: a propensity-matched analysis. Dig Endosc 2019;31:86-93.

6. Shimatani M, Tokuhara M, Kato K, et al. Utility of newly developed short-type double-balloon endoscopy for endoscopic retrograde cholangiography in postoperative patients. 
J Gastroenterol Hepatol 2017;32:1348-1354.

7. Ogura T, Higuchi K. Technical tips for endoscopic ultrasound-guided hepaticogastrostomy. World J Gastroenterol 2016;22:3945-3951.

8. Martins FP, Rossini LG, Ferrari AP. Migration of a covered metallic stent following endoscopic ultrasound-guided hepaticogastrostomy: fatal complication. Endoscopy 2010;42 Suppl 2:E126-E127.

9. De Moura DT, Mestieri LH, Cheng S, et al. Natural orifice transluminal endoscopic surgery to salvage a migrated stent during EUS-guided hepaticogastrostomy. Gastrointest Endosc 2016;83:656-657.

10. Minaga K, Kitano M, Yamashita Y, Nakatani Y, Kudo M. Stent migration into the abdominal cavity after EUS-guided hepaticogastrostomy. Gastrointest Endosc 2017;85:263-264.

11. Kamata K, Takenaka M, Minaga K, et al. Stent migration during EUS-guided hepaticogastrostomy in a patient with massive ascites: troubleshooting using additional EUS-guided antegrade stenting. Arab J Gastroenterol 2017;18:120-121.

12. van Geenen EJM, Siersema PD. Stent migration into the abdominal cavity after EUS-guided hepaticogastrostomy. Gastrointest Endosc 2018;87:617-618.

13. Yang MJ, Kim JH, Kim DJ, Hwang JC, Yoo BM. Hepatobiliary and pancreatic: EUS-guided reintervention for extraluminal stent migration after EUS-guided hepaticogastrostomy. J Gastroenterol Hepatol 2018;33:772.

14. Bories E, Pesenti C, Caillol F, Lopes C, Giovannini M. Transgastric endoscopic ultrasonography-guided biliary drainage: results of a pilot study. Endoscopy 2007;39:287-291.

15. Park DH, Jang JW, Lee SS, Seo DW, Lee SK, Kim MH. EUSguided biliary drainage with transluminal stenting after failed ERCP: predictors of adverse events and long-term results. Gastrointest Endosc 2011;74:1276-1284.

16. Vila JJ, Pérez-Miranda M, Vazquez-Sequeiros E, et al. Initial experience with EUS-guided cholangiopancreatography for biliary and pancreatic duct drainage: a Spanish national survey. Gastrointest Endosc 2012;76:1133-1141.

17. Park DH, Jeong SU, Lee BU, et al. Prospective evaluation of a treatment algorithm with enhanced guidewire manipulation protocol for EUS-guided biliary drainage after failed ERCP (with video). Gastrointest Endosc 2013;78:91-101.

18. Kawakubo K, Isayama H, Kato H, et al. Multicenter retrospective study of endoscopic ultrasound-guided biliary drainage for malignant biliary obstruction in Japan. J Hepatobiliary Pancreat Sci 2014;21:328-334.

19. Paik WH, Park DH, Choi JH, et al. Simplified fistula dilation technique and modified stent deployment maneuver for EUS-guided hepaticogastrostomy. World J Gastroenterol 2014;20:5051-5059.

20. Artifon EL, Marson FP, Gaidhane M, Kahaleh M, Otoch JP. Hepaticogastrostomy or choledochoduodenostomy for distal malignant biliary obstruction after failed ERCP: is there any difference? Gastrointest Endosc 2015;81:950-959.

21. Umeda J, Itoi $\mathrm{T}$, Tsuchiya $\mathrm{T}$, et al. A newly designed plastic stent for EUS-guided hepaticogastrostomy: a prospective preliminary feasibility study (with videos). Gastrointest Endosc 2015;82:390-396.

22. Poincloux L, Rouquette O, Buc E, et al. Endoscopic ultrasound-guided biliary drainage after failed ERCP: cumulative experience of 101 procedures at a single center. Endoscopy 2015;47:794-801.

23. Khashab MA, Messallam AA, Penas I, et al. International multicenter comparative trial of transluminal EUS-guided biliary drainage via hepatogastrostomy vs. choledochoduodenostomy approaches. Endosc Int Open 2016;4:E175-E181.

24. Ogura T, Chiba Y, Masuda D, et al. Comparison of the clinical impact of endoscopic ultrasound-guided choledochoduodenostomy and hepaticogastrostomy for bile duct obstruction with duodenal obstruction. Endoscopy 2016;48:156163.

25. Nakai Y, Isayama H, Yamamoto N, et al. Safety and effectiveness of a long, partially covered metal stent for endoscopic ultrasound-guided hepaticogastrostomy in patients with malignant biliary obstruction. Endoscopy 2016;48:1125-1128.

26. Paik WH, Lee NK, Nakai Y, et al. Conversion of external percutaneous transhepatic biliary drainage to endoscopic ultrasound-guided hepaticogastrostomy after failed standard internal stenting for malignant biliary obstruction. Endoscopy 2017;49:544-548.

27. Minaga K, Takenaka M, Kitano M, et al. Rescue EUS-guided intrahepatic biliary drainage for malignant hilar biliary stricture after failed transpapillary re-intervention. Surg Endosc 2017;31:4764-4772.

28. Ogura T, Onda S, Takagi W, et al. Clinical utility of endoscopic ultrasound-guided biliary drainage as a rescue of reintervention procedure for high-grade hilar stricture. J Gastroenterol Hepatol 2017;32:163-168.

29. Cho DH, Lee SS, Oh D, et al. Long-term outcomes of a newly developed hybrid metal stent for EUS-guided biliary drainage (with videos). Gastrointest Endosc 2017;85:10671075.

30. Sportes A, Camus M, Greget M, et al. Endoscopic ultrasound-guided hepaticogastrostomy versus percutaneous transhepatic drainage for malignant biliary obstruction after failed endoscopic retrograde cholangiopancreatography: a retrospective expertise-based study from two centers. Therap Adv Gastroenterol 2017;10:483-493.

31. Moryoussef F, Sportes A, Leblanc S, Bachet JB, Chaussade $\mathrm{S}$, Prat F. Is EUS-guided drainage a suitable alternative technique in case of proximal biliary obstruction? Therap Adv Gastroenterol 2017;10:537-544.

32. Oh D, Park DH, Song TJ, et al. Optimal biliary access point 
and learning curve for endoscopic ultrasound-guided hepaticogastrostomy with transmural stenting. Therap Adv Gastroenterol 2017;10:42-53.

33. Honjo M, Itoi T, Tsuchiya T, et al. Safety and efficacy of ultra-tapered mechanical dilator for EUS-guided hepaticogastrostomy and pancreatic duct drainage compared with electrocautery dilator (with video). Endosc Ultrasound 2018;7:376-382.

34. Okuno N, Hara K, Mizuno N, et al. Efficacy of the 6-mm fully covered self-expandable metal stent during endoscopic ultrasound-guided hepaticogastrostomy as a primary biliary drainage for the cases estimated difficult endoscopic retrograde cholangiopancreatography: a prospective clinical study. J Gastroenterol Hepatol 2018;33:1413-1421.

35. Miyano A, Ogura T, Yamamoto K, Okuda A, Nishioka N, Higuchi K. Clinical impact of the intra-scope channel stent release technique in preventing stent migration during EUS-guided hepaticogastrostomy. J Gastrointest Surg 2018;22:1312-1318.

36. Paik WH, Lee TH, Park DH, et al. EUS-guided biliary drainage versus ERCP for the primary palliation of malignant biliary obstruction: a multicenter randomized clinical trial. Am J Gastroenterol 2018;113:987-997.

37. Kanno Y, Koshita S, Ogawa T, et al. EUS-guided biliary drainage for unresectable malignant biliary obstruction: 10year experience of 99 cases at a single center. J Gastrointest Cancer 2019;50:469-477.

38. Minaga K, Ogura T, Shiomi H, et al. Comparison of the efficacy and safety of endoscopic ultrasound-guided choledochoduodenostomy and hepaticogastrostomy for malignant distal biliary obstruction: multicenter, randomized, clinical trial. Dig Endosc 2019;31:575-582.

39. Ogura T, Okuda A, Miyano A, et al. EUS-guided versus percutaneous biliary access in patients with obstructive jaundice due to gastric cancer. Dig Liver Dis 2019;51:247-252.

40. Kaneko J, Ishiwatari H, Takizawa $\mathrm{K}$, et al. Mediastinitis due to perforation by a metal stent after endoscopic ultrasoundguided hepaticogastrostomy: a rare complication. Endoscopy 2020;52:519-521.

41. Ogura T, Yamada M, Nishioka N, Yamada T, Higuchi K.
Gastrointestinal: knuckle guidewire insertion: safe techniques of guidewire insertion into the pancreatobiliary tract using a novel 0.025-inch guidewire. J Gastroenterol Hepatol 2020;35:707.

42. Ogura T, Masuda D, Takeuchi T, Fukunishi S, Higuchi K. Liver impaction technique to prevent shearing of the guidewire during endoscopic ultrasound-guided hepaticogastrostomy. Endoscopy 2015;47:E583-E584.

43. Ryou M, Benias PC, Kumbhari V. Initial clinical experience of a steerable access device for EUS-guided biliary drainage. Gastrointest Endosc 2020;91:178-184.

44. Amano M, Ogura T, Onda S, et al. Prospective clinical study of endoscopic ultrasound-guided biliary drainage using novel balloon catheter (with video). J Gastroenterol Hepatol 2017;32:716-720.

45. Ogura T, Nakai Y, Iwashita T, Higuchi K, Itoi T. Novel fine gauge electrocautery dilator for endoscopic ultrasoundguided biliary drainage: experimental and clinical evaluation study (with video). Endosc Int Open 2019;7:E1652-E1657.

46. Park DH, Lee TH, Paik WH, et al. Feasibility and safety of a novel dedicated device for one-step EUS-guided biliary drainage: a randomized trial. J Gastroenterol Hepatol 2015;30:1461-1466.

47. Ogura T, Yamamoto K, Sano T, et al. Stent length is impact factor associated with stent patency in endoscopic ultrasound-guided hepaticogastrostomy. J Gastroenterol Hepatol 2015;30:1748-1752.

48. Hamada T, Nakai Y, Isayama H, Koike K. Tandem stent placement as a rescue for stent misplacement in endoscopic ultrasonography-guided hepaticogastrostomy. Dig Endosc 2013;25:340-341.

49. Ogura T, Kitano M, Takenaka M, et al. Multicenter prospective evaluation study of endoscopic ultrasound-guided hepaticogastrostomy combined with antegrade stenting (with video). Dig Endosc 2018;30:252-259.

50. Minaga K, Takenaka M, Miyata T, Ueda Y, Kitano M, Kudo M. Through-the-mesh technique after endoscopic ultrasonography-guided hepaticogastrostomy: a novel reintervention method. Endoscopy 2016;48:E369-E370. 